a human monoclonal antibody directed against the IL- 6 receptor- $\alpha$ (TARGET [NCT01709578]; MONARCH [NCT02332590]).

Objectives: To evaluate patient-perceived impact of sarilumab on RA using the RAID scale vs either placebo + conventional synthetic disease-modifying anti-rheumatic drugs (csDMARDs) or adalimumab.

Methods: TARGET assessed sarilumab $150 \mathrm{mg}$ and $200 \mathrm{mg}$ added to csDMARDs vs placebo in patients with RA intolerant of or not responding to anti-TNF therapy. MONARCH assessed sarilumab $200 \mathrm{mg}$ monotherapy vs adalimumab $40 \mathrm{mg}$ monotherapy in patients with RA either intolerant of, inadequate responders to, or considered inappropriate candidates for continued treatment with methotrexate. Treatments were administered subcutaneously every 2 weeks. RAID has 7 single-item domains, each rated by patients on an 11-point numeric rating scale from 0 (absence) to 10 (extreme). A total score from 0 to 10 (with lower scores indicative of less impact of disease) is calculated by weighting responses for each item based on patient assessment of the relative importance of the item. RAID was assessed at baseline (BL), Weeks 12 and 24. Least square mean (LSM) changes from BL in total score (Weeks 12 and 24) and domains (Week 24 only) were analysed with a mixed model for repeated measures, including treatment, region, visit, and treatment-by-visit interaction (and prior csDMARD therapy in TARGET) as fixed effects and BL as a covariate. RAID was tested outside of trial hierarchy and statistical significance is not claimed; nominal p-values are provided. Post-hoc categorical change analyses were conducted to identify "responders" in the total score (improvements $\geq$ minimum clinically important difference from BL to Week 24 [absolute change of 3 or relative change of $50 \%$ in total score]). Patients discontinuing therapy/requiring rescue medication prior to endpoint were classified as non-responders.

Results: Sarilumab was superior (nominal $\mathrm{p}<0.05$ ) to placebo (TARGET) and adalimumab (MONARCH) at Weeks 12 and 24 for RAID total score (Table). There was a greater proportion of responders in both sarilumab dose groups vs placebo at both time points (TARGET) and in sarilumab $200 \mathrm{mg}$ vs adalimumab at Week 24 (MONARCH). The effect of sarilumab was consistent across all 7 individual RAID domains (nominal $p<0.05$ ) at Week 24, except for sleep difficulties vs placebo in TARGET. Effects of placebo were highest on pain and effects of sarilumab were lowest on emotional well-being (TARGET) and coping (MONARCH)

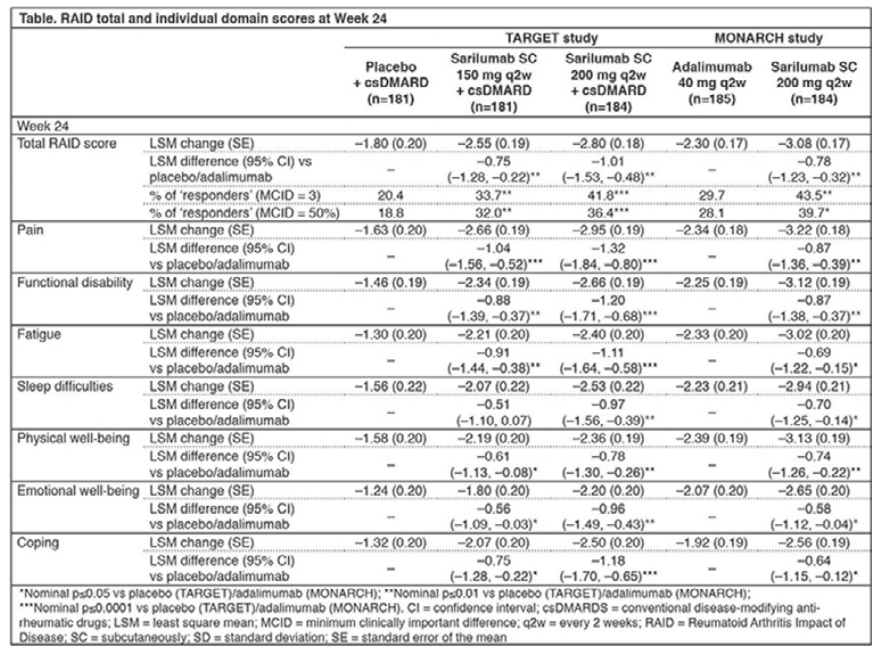

Conclusions: Assessed using RAID, sarilumab either with csDMARDs or as monotherapy reduced the impact of RA on patients' lives to a greater extent than placebo+csDMARDs or adalimumab monotherapy, with benefits shown on total RAID and all 7 individual domain scores.

Acknowledgements: This study was sponsored by Sanofi and Regeneron Pharmaceuticals, Inc.

Disclosure of Interest: L. Gossec Consultant for: Abbvie, Celgene, Janssen, Lilly, Novartis, MSD, Roche, and UCB, V. Strand Consultant for: AbbVie, Amgen, AstraZeneca, Biogen, BMS, Celltrion, CORRONA, Crescendo, Genentech/Roche, GSK, Janssen, Eli Lilly, Novartis, Pfizer, Regeneron Pharmaceuticals, Sandoz, Sanofi, and UCB, C. Proudfoot Shareholder of: Sanofi, Employee of: Sanofi, C. Chen Shareholder of: Regeneron Pharmaceuticals, Inc, Employee of: Regeneron Pharmaceuticals, Inc, S. Guillonneau Shareholder of: Sanofi, Employee of: Sanofi, T. Kimura Shareholder of: Regeneron Pharmaceuticals, Inc, Employee of: Regeneron Pharmaceuticals, Inc, H. van Hoogstraten Shareholder of: Sanofi, Employee of: Sanofi, E. Mangan Shareholder of: Regeneron Pharmaceuticals, Inc, Employee of: Regeneron Pharmaceuticals, Inc, M. Reaney Shareholder of: Sanofi, Employee of: Sanofi DOI: 10.1136/annrheumdis-2017-eular.3448

\section{FRI0241 THE EFFECT OF ABATACEPT ON CYTOKINE PROFILE IN PATIENTS WITH RHEUMATOID ARTHRITIS}

M.A. Borisova, G.V. Lukina, Y. Sigidin, E.L. Luchihina, D.E. Karateev, A. Novikov, E. Alexandrova, E. Aronova, S. Glukhova, E. Nasonov. Nasonova Reserch Institute of Rheumatology, MOSCOW, RUSSIA, Moscow, Russian Federation

Background: Pathological activation of T-cells with the overproduction of proinflammatory cytokines is playing a major role in the pathogenesis of rheumathoid arthritis (RA). The influence of the selective co-stimulation modulator abatacept (ABA) on the dynamics of cytokine profile in patients with RA is not fully understood.

Objectives: To assess the changes in cytokine profile in patients treated with ABA.

Methods: 44 patients with RA and an inadequate response to synthetic DMARDs or biologics were enrolled in the study. Most of them were middle aged females (46,9 $\pm 13,9$ years) with median RA duration 2 years $(1,4-3)$, high disease activity (DAS28 $=5,2 \pm 0,8)$, RF-positive $(80 \%)$ and ACPA-positive (79,5\%). 16 healthy individuals were included in the study as control. The serum levels of IL-1, IL-6, IL-17, TNF- $\alpha$, VEGF, IP-10 (pg/ml) were measured by ELISA immunoassay, YKL-40 by MicroVue immunoassay at baseline and 24 weeks. Disease activity was measured by DAS28, results were assessed every 12 weeks by EULAR criteria. ABA was administered intravenously every 4 weeks.

Results: Levels of IL-6 $(2.4(1,1-6,4)$ vs $0.7(0,62-1,0), p=0,0002)$, YKL-40 (97 $(68,4-97,9)$ vs. $64(52,4-107,5), \mathrm{p}=0,03), \mathrm{IP}-10(21(12,9-49,8)$ vs $14(9,2-15,2)$, $p=0,005$ ) were significantly higher in patients with $R A$ compared to control. $A B A$ significant reduced disease activity already after 12 weeks of therapy $(p<0,05)$. After 24 weeks of ABA therapy good and moderate response by EULAR criteria was achieved in $86 \%$, low disease activity by DAS28 in $52 \%$. By the 6 -th month ABA significant decreased levels of IL-6 $(1,29(0,9-2,2, p=0,0006)$, IP-10 (14 $(7,5-28), p=0.007)$ as well as MMP3: before 30.1 (13-82), after 24 weeks 10 (7.4-55), $\mathrm{p}=0.0003$ and RF: before 218 (9.6-187), after 24 weeks 159 (9.7-155), $\mathrm{p}=0.02$. Lowering of the IL-6 $(r=0,5)$ and IP-10 $(r=0,32)$ levels were significantly $(p<0.05)$ associated with a decrease of DAS28.

Conclusions: ABA therapy leads to a significant reduction in serum levels of IL-6, IP-10, MMP3 and RF. The serum levels of IL-6 and IP-10 correlate with decrease activity of RA.

Disclosure of Interest: None declared

DOI: 10.1136/annrheumdis-2017-eular.6540

\section{FRI0242 ASSOCIATION BETWEEN CLINICAL AND RADIOGRAPHIC RESPONSES, AND PHYSICAL FUNCTION IN A PHASE 3 STUDY OF SARILUMAB PLUS METHOTREXATE IN PATIENTS WITH ACTIVE, MODERATE-TO-SEVERE RHEUMATOID ARTHRITIS}

M.C. Genovese ${ }^{1}$, H. van Hoogstraten ${ }^{2}$, W. Kampman ${ }^{3}$, S. Jayawardena ${ }^{2}$,

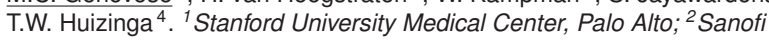
Genzyme, Bridgewater; ${ }^{3}$ Regeneron Pharmaceuticals, Inc., Tarrytown, United States; ${ }^{4}$ Leiden University Medical Centre, Leiden, Netherlands

Background: In MOBILITY (NCT01061736), SC sarilumab (150 or 200 mg q2w) + MTX demonstrated efficacy in adults with RA and inadequate response to MTX. The most common TEAEs were infections, neutropenia, injection site reactions, and increased transaminases.

Objectives: To examine association between clinical response and radiographic progression and functional response (HAQ-DI) in MOBILITY.

Methods: In this post hoc analysis, associations between HAQ-DI and clinical efficacy categories (CDAI, DAS28-CRP, SDAl, and Boolean-based ACR/EULAR remission) were tested at wk 16. Trend for change from baseline (BL) in HAQ-DI across response categories was assessed using the Jonckheere-Terpstra test

Results: Regardless of definition, percentage achieving remission (CDAI $\leq 2.8$, DAS28-CRP $<2.6$, SDAl $<3.3$ ) or no $x$-ray progression was higher with sarilumab vs $\mathrm{Pbo}(P<0.05)$. Overall, there was a significant trend between magnitude of clinical response at wk 16 and improvement in physical function (Table). This trend was also observed for radiographic progression (mTSS change from $\mathrm{BL}$ ),

\begin{tabular}{|c|c|c|c|c|c|c|c|c|}
\hline & \multicolumn{2}{|c|}{$\begin{array}{c}P b o+M T X \\
N=398\end{array}$} & \multicolumn{2}{|c|}{$\begin{array}{c}\text { Sarilumab } \\
150 \underset{\mathrm{mg} \mathrm{q}}{\mathrm{N}=400}+\mathrm{MTX}\end{array}$} & \multicolumn{2}{|c|}{$\begin{array}{c}\text { Sarilumab } \\
200 \underset{\mathrm{mg} \mathrm{q}}{\mathrm{N}=399}+\mathrm{MTX}\end{array}$} & \multicolumn{2}{|c|}{$\begin{array}{c}\text { All patients* } \\
\mathrm{N}=1197\end{array}$} \\
\hline & $n(\%)$ & mean (SD) & $\mathbf{n}(\%)$ & mean (SD) & $n(\%)$ & mean $(\mathrm{SD})$ & $n(\%)$ & mean (SD) \\
\hline \multicolumn{9}{|l|}{ CDAI } \\
\hline$\leq 2.8$ & $13(3)$ & $-0.7(0.5)$ & $25(7)^{\dagger}$ & $-0.8(0.6)$ & $40(11)^{1}$ & $-1.1(0.7)$ & $78(7)$ & $-0.9(0.7)$ \\
\hline$>2.8-\leq 10$ & $47(12)$ & $-0.8(0.8)$ & $92(23)$ & $-0.7(0.6)$ & $105(26)$ & $-0.8(0.6)$ & $244(20)$ & $-0.7(0.6)$ \\
\hline$>10$ & $315(79)$ & $.0 .2(0.5)$ & $242(61)$ & $-0.5(0.5)$ & $218(55)$ & $.0 .4(0.5)$ & $775(65)$ & $-0.3(0.5)$ \\
\hline \multicolumn{9}{|c|}{ DAS28-CRP } \\
\hline$<2.6$ & $22(6)$ & $-0.7(0.6)$ & $88(25)^{\top}$ & $-0.8(0.6)$ & $109(31)^{1}$ & $-0.9(0.7)$ & $219(18)$ & $-0.8(0.6)$ \\
\hline$\geq 2.6-\leq 3.2$ & $30(8)$ & $-0.6(0.9)$ & $59(17)$ & $-0.6(0.6)$ & $66(19)$ & $-0.5(0.5)$ & $155(14)$ & $-0.6(0.6)$ \\
\hline$>3.2-<5.1$ & 154 (41) & $-0.4(0.5)$ & $149(42)$ & $-0.5(0.5)$ & $149(42)$ & $.0 .4(0.6)$ & $450(38)$ & $-0.4(0.5)$ \\
\hline$>5.1$ & $168(45)$ & $-0.1(0.5)$ & $61(17)$ & $-0.3(0.5)$ & $30(8)$ & $.0 .2(0.6)$ & $259(24)$ & $-0.2(0.5)$ \\
\hline \multicolumn{9}{|c|}{ X-ray progression ( $\leq 0$ cutoff) } \\
\hline No & $154(39)$ & $-0.3(0.5)$ & $191(48)^{\dagger}$ & $-0.5(0.6)$ & $222(56)^{1}$ & $.0 .6(0.6)$ & $550(46)$ & $-0.5(0.6)$ \\
\hline Yes & $244(61)$ & $-0.3(0.6)$ & $209(52)$ & $-0.6(0.5)$ & $177(44)$ & $-0.5(0.6)$ & $555(46)$ & $-0.4(0.6)$ \\
\hline \multicolumn{9}{|c|}{ X-ray progression ( 50.5 cutoff) } \\
\hline No & $172(43)$ & $-0.4(0.5)$ & $219(55)^{1}$ & $-0.5(0.5)$ & $257(64)^{7}$ & $-0.6(0.6)$ & 648 (54) & $-0.5(0.6)$ \\
\hline Yes & 226 (57) & $-0.2(0.6)$ & $181(45)$ & $-0.6(0.5)$ & $142(36)$ & $-0.4(0.6)$ & $549(46)$ & $-0.4(0.6)$ \\
\hline
\end{tabular}


regardless of cutoff $(\leq 0.5$ and $\leq 0)$. In patients achieving remission, there was a numerically greater improvement in HAQ-DI with sarilumab vs placebo (Pbo). Even if patients did not achieve remission or LDA, the sarilumab group had generally greater numerical improvements in $\mathrm{HAQ}-\mathrm{DI}$ vs $\mathrm{Pbo}$.

Conclusions: Achieving LDA or remission, or absence of radiographic progression, was associated with overall greater improvement in physical function. Irrespective of whether patients achieved remission or LDA, sarilumab + MTX showed greater improvements in HAQ-DI than Pbo + MTX.

Acknowledgements: This study was sponsored by Sanofi Genzyme and Regeneron Pharmaceuticals, Inc. Editorial support was provided by MedThink SciCom and funded by Sanofi Genzyme and Regeneron Pharmaceuticals, Inc. Disclosure of Interest: M. Genovese Grant/research support from: Roche, Sanofi, GlaxoSmithKline, R-Pharma, RuiYi, and Bristol-Myers Squibb, Consultant for: Roche, Sanofi, GlaxoSmithKline, R-Pharma, RuiYi, and Bristol-Myers Squibb, H. van Hoogstraten Shareholder of: Sanofi Genzyme, Employee of: Sanofi Genzyme, W. Kampman Shareholder of: Regeneron Pharmaceuticals, Inc, Employee of: Regeneron Pharmaceuticals, Inc, S. Jayawardena Shareholder of: Sanofi Genzyme, Employee of: Sanofi Genzyme, T. Huizinga Consultant for: Sanofi, Roche, and Abblynx.

DOI: 10.1136/annrheumdis-2017-eular.3513

\section{FRI0243 SIRUKUMAB TREATMENT REDUCES LEVELS OF IRON-REGULATORY PROTEINS AND AMELIORATES INFLAMMATION-ASSOCIATED ANEMIA IN RHEUMATOID ARTHRITIS PATIENTS}

M. Loza ${ }^{1}$, K. Campbell ${ }^{1}$, K. Sweet ${ }^{1}$, B. Hsu ${ }^{1}$, S. Daga ${ }^{2}$, B. Dasgupta ${ }^{1}$ ${ }_{1}^{1}$ Janssen Research \& Development, LLC, Spring House, PA, United States;

${ }^{2}$ GlaxoSmithKline, Uxbridge, United Kingdom

Background: Anemia of chronic disease, a common comorbidity of rheumatoid arthritis (RA), is detrimental to patients' (pts) quality of life, productivity, and long-term health.

Objectives: Sirukumab (SIR), a human monoclonal antibody that selectively binds to the IL-6 cytokine with high affinity, has recently demonstrated efficacy in RA. It was hypothesized that SIR, compared to placebo (pbo) and tumor necrosis factor-inhibitors (TNFi), increases hemoglobin $(\mathrm{Hb})$ concentrations in RA pts by decreasing levels of iron-scavenging proteins and increasing transferrin levels, thus ameliorating anemia. This hypothesis was tested in post hoc analyses of 4 phase 3 studies of SIR in RA: SIRROUND-M (methotrexate [MTX] inadequate responders [IR]); -D (disease-modifying antirheumatic drug [DMARD] IR); -T (TNFi-IR); - H (MTX-IR, monotherapy).

Methods: Standard hematology measurements, including $\mathrm{Hb}$ levels, were made throughout the studies by a central laboratory. Anemia was defined as $\mathrm{Hb}$ levels $<125 \mathrm{~g} / \mathrm{L}$ (males) and $<115 \mathrm{~g} / \mathrm{L}$ (females). In a subset of pts, iron-regulatory proteins (hepcidin, haptoglobin, hemopexin, transferrin) were measured in serum at baseline (BL) and Wk 4 using the SomaLogic SOMAscan ${ }^{\mathrm{TM}}$ platform (for SIRROUND-M, -D, -T, and -H studies, respectively: SIR 100mg q2w, $\mathrm{n}=61,205$, 136, 0; SIR 50mg q4w, $n=61,201,128,100$; pbo, $n=0,118,56,0$; adalimumab [ADA], $\mathrm{n}=0,0,0,98$ )

Results: SIR consistently reduced the prevalence of anemia to a greater extent than was observed for pbo $(p<0.05$; eg, in SIRROUND-D, anemia decreased from $25 \%$ of pts at $\mathrm{BL}$ to $10 \%$ at Wk 16 post-treatment with SIR $50 \mathrm{mg}$ q $4 \mathrm{w}$ vs increase from $24 \%$ of pts to $28 \%$ with pbo) and ADA (SIRROUND-H). Across studies on SIR $50 \mathrm{mg} \mathrm{q} 4 \mathrm{w}$, increases in $\mathrm{Hb}$ levels ranged from $7 \pm 10$ to $10 \pm 11 \mathrm{~g} / \mathrm{L}$ (mean $\pm S D$ ) through Wk 16; however, greater changes were seen in pts anemic at $\mathrm{BL}(13 \pm 13$ to $16 \pm 12 \mathrm{~g} / \mathrm{L}$ increase). Significant $\mathrm{Hb}$ elevations were observed by Wk 2, with comparable results for SIR $100 \mathrm{mg}$ q2w. Statistically significant increases in $\mathrm{Hb}$ levels were not observed with ADA (Fig.1) or pbo, regardless of $\mathrm{BL}$ anemia status. Changes in Hb levels with SIR were independent of changes in RA disease activity. Mean haptoglobin levels were modestly higher at $B L$ in RA pts with anemia compared to pts without anemia. Across studies, both SIR doses similarly strongly decreased levels of hepcidin, haptoglobin, and hemopexin and increased transferrin levels at Wk 4, regardless of $\mathrm{BL}$ anemia status. The modulation of these proteins by ADA was considerably less (Fig.1) and by pbo non-significant. After SIR treatment, greater decreases in hepcidin levels were consistently observed in pts with vs without BL anemia across studies by Wk 4 .
A

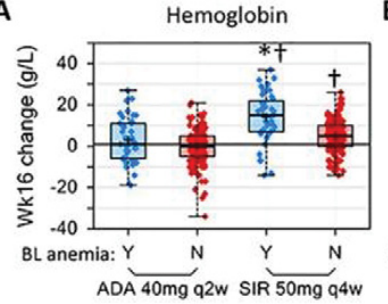

B

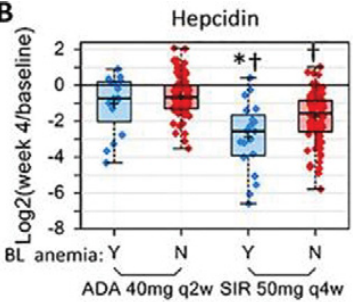

Figure 1. For the SIRROUND-H study, changes in (A) hemoglobin (week 16 change from baseline in $\mathrm{g} / \mathrm{L}$ ) and (B) hepcidin ( $\log _{2}$ (week $4 /$ baseline)) are plotted for each available patient, stratified by treatment group and baseline (BL) anemia status ( $\mathrm{Y}$, blue; $\mathrm{N}$, red). ${ }^{*} \mathrm{p}<0.05$ for baseline anemia $Y$ vs. $N$ within treatment group; $+p<0.05$ vs. adalimumab, within baseline anemia group. ADA, adalimumab; SIR, sirukumab.
Conclusions: SIR consistently increased Hb levels in RA pts (DMARD-IR, TNFi$I R$, monotherapy), most prominently in pts with $B L$ anemia, resulting in significant reductions in the prevalence of anemia. These effects were independent of the extent of improvement in RA disease activity, suggesting additional benefits of SIR beyond clinical response in RA. By inhibiting IL-6, SIR may decrease key iron-regulatory proteins, such as hepcidin, and shift homeostasis towards an increase in the pool of iron available for red blood cell $\mathrm{Hb}$, thus ameliorating anemia of chronic inflammation associated with RA.

Disclosure of Interest: M. Loza Employee of: Janssen Research \& Development, LLC, K. Campbell Employee of: Janssen Research \& Development, LLC, K. Sweet Employee of: Janssen Research \& Development, LLC, B. Hsu Shareholder of: Janssen Research \& Development, LLC, Employee of: Janssen Research \& Development, LLC, S. Daga Shareholder of: GlaxoSmithKline, Employee of: GlaxoSmithKline, B. Dasgupta Employee of: Janssen Research \& Development, LLC

DOI: 10.1136/annrheumdis-2017-eular.3570

\section{FRI0244 LOW DOSE OF RITUXIMAB IS EFFECTIVE FOR MAINTENANCE OF CLINICAL REMISSION OR LOW DISEASE ACTIVITY IN PATIENTS WITH RHEUMATOID ARTHRITIS}

N. Avgoustidis, I. Flouri, C. Adamichou, E. Kabouraki, A. Eskitzis, P. Kyfonidou, I. Papalopoulos, A. Fanouriakis, A. Repa, N. Kougkas, G. Bertsias,

P. Sidiropoulos. Department of Rheumatology, Clinical Immunology and Allergy, University Hospital of Heraklion, Crete, Greece, Heraklion, Greece

Background: Although existing data support the effectiveness of the low dose regimen (LDR, 1gr every 6 months) of rituximab as initial treatment for active rheumatoid arthritis (RA) (2), it is unknown whether this regimen may be used to maintain the therapeutic effect in patients achieved clinical remission or low disease activity (LDA) upon treatment with conventional rituximab regimen (2gr every 6 months)

Objectives: To evaluate the effectiveness of the rituximab LDR for maintenance of clinical remission and/or LDA in patients with RA in clinical practice.

Methods: Long-term prospective study of RA patients who received rituximab in the Rheumatology Department of the University Hospital of Heraklion during 03/2005-07/2016. All patients on clinical remission [DAS 28 (ESR) <2.6] or LDA [DAS28 (ESR) <3.2] for at least 12 months were treated with the LDR after obtaining verbal consent

Results: We analyzed 247 patients who received conventional rituximab regimen, of median age (IQR) 62 (54.3-70.4)years, females (84\%), disease duration 9.6 (5.6-18.3) years, $52 \%$ seropositive (RF or anti-CCP). Patients have had failed in (median) $3(2-3)$ non-biologic (nbDMARDs) and $1(1-2)$ biologic DMARDs (bDMARDs) before rituximab initiation. At baseline of rituximab treatment, $58.3 \%$ and $91.1 \%$ of them were on steroids and (nbDMARDs) respectively, while the disease activity was high [mean DAS28 (ESR): 5.84 (5.20-6.49)] and they had impaired physical functioning [mean HAQ: $1.0(0.63-1.38)$ ].

Overall, 27/247patients (11\%) received the LDR. Before the initiation of LDR, the duration of rituximab treatment was 24 (18-48) months and cumulative rituximab "exposure" was 8 (6-15) gr. At the time of LDR initiation, disease activity [DAS28 (ESR)] was $2.8(2.2-3.6)$ and HAQ: $0(0-0.4)$, while the time needed for achieving remission or LDA was18 (8-23) months. The median duration of follow-up of patients on LDR was $12(6-20)$ months. $23(85 \%)$ of patients remained in remission or LDA with median DAS28 (ESR) $2.85(2.23-3.52)$ and HAQ 0 (0-0.5) at last follow-up. Only $3(11 \%)$ of the patients experienced an increase of DAS28 $(>1.2)$ and $2(7 \%)$ of patients return to conventional dose.

Conclusions: In clinical practice, RA patients who achieved remission or low disease activity with conventional dose of rituximab may sustain clinical responses if treated with LDR. These preliminary findings support the use of LDR as maintenance treatment regimen and this may allow cost savings. (1)

References:

[1] Gianfranco Ferraccioli et al.Could we use a lower dose of rituximab to treat rheumatoid arthritis in clinical practice: pros and cons? Arthritis Res Ther. 2016; 18: 126

[2] Chatzidionysiou K, et al. Effectiveness of two different doses of Rituximab for the treatment of rheumatoid arthritis in an international cohort: data from the CERERRA collaboration. Arthritis Res Ther. 2016; 18:50.

Disclosure of Interest: None declared

DOI: 10.1136/annrheumdis-2017-eular.4958

\section{FRI0245 ABATACEPT RETENTION RATES AND PROGNOSTIC FACTORS OF RETENTION IN PATIENTS WITH RHEUMATOID ARTHRITIS: 2-YEAR RESULTS FROM THE REAL-WORLD ACTION STUDY}

$\underline{\text { R. Alten }}{ }^{1}$, H.-M. Lorenz ${ }^{2}$, X. Mariette ${ }^{3}$, H. Nüßlein ${ }^{4}$, M. Galeazzi ${ }^{5}$, F. Navarro ${ }^{6}$, M. Chartier ${ }^{7}$, Y. Elbez ${ }^{8}$, C. Rauch ${ }^{9}$, M. Le Bars ${ }^{7}$. ${ }^{1}$ Schlosspark-Klinik University Medicine, Berlin; ${ }^{2}$ University Hospital, Heidelberg, Germany; ${ }^{3}$ Université Paris-Sud, Paris, France; ${ }^{4}$ University of Erlangen-Nuremberg, Nuremberg, Germany; ${ }^{5}$ University of Siena, Siena, Italy; ${ }^{6}$ Hospital Universitario Virgen Macarena, Seville, Spain; ${ }^{7}$ Bristol-Myers Squibb, Rueil-Malmaison; ${ }^{8}$ Excelya, Boulogne-Billancourt, France; ${ }^{9}$ Bristol-Myers Squibb, Munich, Germany

Background: The ACTION (NCT02109666) study was designed to provide 